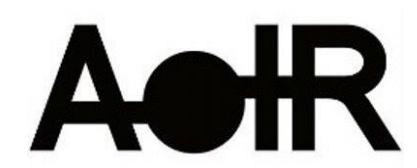

Selected Papers of \#AoIR2021:

The 22nd Annual Conference of the

Association of Internet Researchers

Virtual Event / 13-16 Oct 2021

\title{
MAPPING DISCORD'S DARKSIDE: DISTRIBUTED HATE NETWORKS ON DISBOARD
}

\author{
Daniel G. Heslep \\ University of Alabama \\ PS Berge \\ University of Central Florida
}

\section{Introduction}

Scholars have noted the troubled legacy of Discord, a voice/video community platform, which harbored the white supremacist groups behind the 2017 "Unite the Right" rally (Brown Jr. \& Hennis, 2019). In response to critical attention by journalists and scholars, Discord intensified its moderating practices, and began rebranding itself with an expansion of in-house "trust and safety" and a public commitment to proactive moderation (Discord Transparency Report, n.d.).

While Discord has committed to increased moderation, we question this commitment when Discord does not acknowledge the role of third-party bulletin sites that serve as the interconnective structure of Discord communities. We ask how Discord's continued reliance on third-party services reifies an "outsourcing of responsibility" and facilitates the continuation of white supremacist publics (Brown Jr. \& Hennis, 2019).

Because Discord only allows users to search partnered and verified servers (generally with 10,000 member minimums), third-party sites like Disboard-a public bulletin for Discord servers-are popular for searching smaller communities. Disboard relies on its own third-party bot, which, when integrated directly into Discord servers, displays a public invite link and the number of currently active members (Figure 1). To excavate the role that these third-party services play in facilitating hateful content, our study has two parts:

1. We use Brock's model for critical technoculture discourse analysis (2018) to demonstrate how Discord's rhetorical rebranding and institution of a curated search stratifies their platform: on the surface, they present an idyllic, engineered 
community of vetted servers for incoming users, while obscuring a persistent network of toxic servers beneath.

2. We argue that Discord's commitment to proactive moderation is subverted by its reliance on third-party sites. We demonstrate how third-party structures maintain an aggressively racist and toxic web that underlies the platform. We examine data scraped from 3,600 Discord servers publicly listed on Disboard that actively marked their associations with Nazism, white power, raiding, queerphobia, and toxicity.

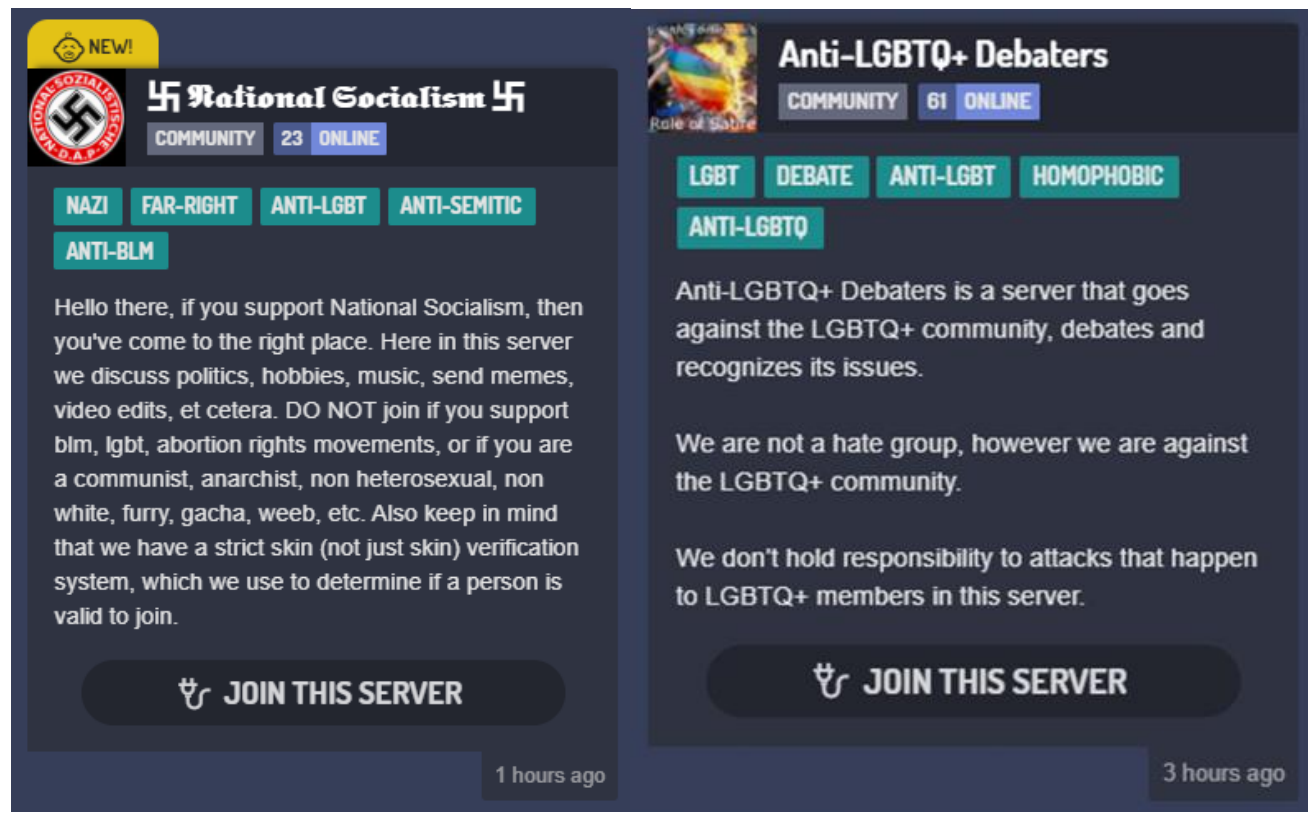

Figure 1. Disboard servers openly promoting hateful affiliations.

\section{Method}

Due to its closed-structure and novelty, research on Discord is nascent. Following the model of work that explores networked harassment (Burgess \& Matamoros-Fernández, 2016), data was scraped from public Discord server bulletin site Disboard

(DiscordFederation/DisboardScraper, 2019/2020). We identified 20+ tags connected to hateful content and scraped data (server name, current online users, description, and 15 tags) for the first 10 pages of servers using each tag. We combined duplicates and used Orange data mining tools and AntConc concordance tools for quantitative analysis.

Because Disboard provides public server descriptions, we were able to ethically collect data about 'toxic' (Massanari, 2017) Discord servers. This work advances scholarship on platform moderation (Gillespie, 2018), and hateful content operating across intraplatform structures (Massanari, 2017). Ultimately, we hope to demonstrate new avenues for researching Discord communities, while engaging in the digital feminist practice of exposing hateful social media structures (D'Ignazio \& Klein, 2020). 


\section{Findings}

We found thousands of Discord servers that marketed themselves on Disboard as hateful and Nazi-affiliated spaces. Disboard's search algorithms exacerbate this linkage, providing recommendations for racist searches that "both informs and is informed in part by users" (Noble, 2018, p. 25; Figure 2).

\section{DISCORD SERVERS}

\section{RACIST}

\section{TAGS SIMILAR TO RACIST}

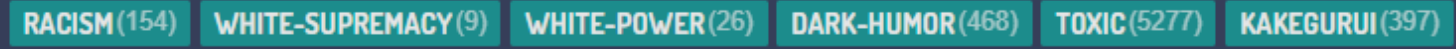

Figure 2. Disboard indexes tags based on racist searches.

In some explicit cases, servers used names (ex. "Hitler's Holy Crusade") and tags (ex. "toxic" [n=3514], "edgy" [n=2363] and "4chan" [n=702]) to market their communities. We identified other alt-right, Nazi, and hate-groups through:

- Use of seemingly innocuous tags such as "Political" or "Roblox" in conjunction with white supremacist tags (e.g. "1488").

- "Conscription servers" established as Nazi recruitment checkpoints, vetting users for private servers.

- Antagonistic servers organized around queerphobia (ex. "anti-lgbt" [n=169]) and toxic-geek masculine discourse (ex. "anti-furry" [n=644], "anti-gacha" [n=308]).

This network of servers-some with hundreds of active users-indicates the presence of systemic, hateful structures. These servers advertised the very tools that Discord has promoted as part of its approach to user safety (Safety Principles, n.d.). Many servers claimed to use Discord's phone-based verification systems and new-member vetting systems to prevent "degenerates" from infiltrating their communities. Additionally, they advertised the way that third-party bots operated in their communities: one server boasted about a third-party bot called " $n$-word deleter" that "runs every $24 \mathrm{~h}$ so your account is safe with us!" While bots are an essential part of moderation for Discord servers (Jiang et al., 2019), these servers actively abuse these tools to encourage harassment. 


\section{Conclusion}

That these hateful structures exist is less important for Discord than if they exist visibly - and herein lies the financial impetus for Discord to continue to allow sites like Disboard to do its dirty work, as it maintains a presentable image for its ongoing expansion. Although Discord's in-house search promotes only curated servers, it has allowed third-party services to imitate its user interface (Figure 3), and network hate on its behalf.

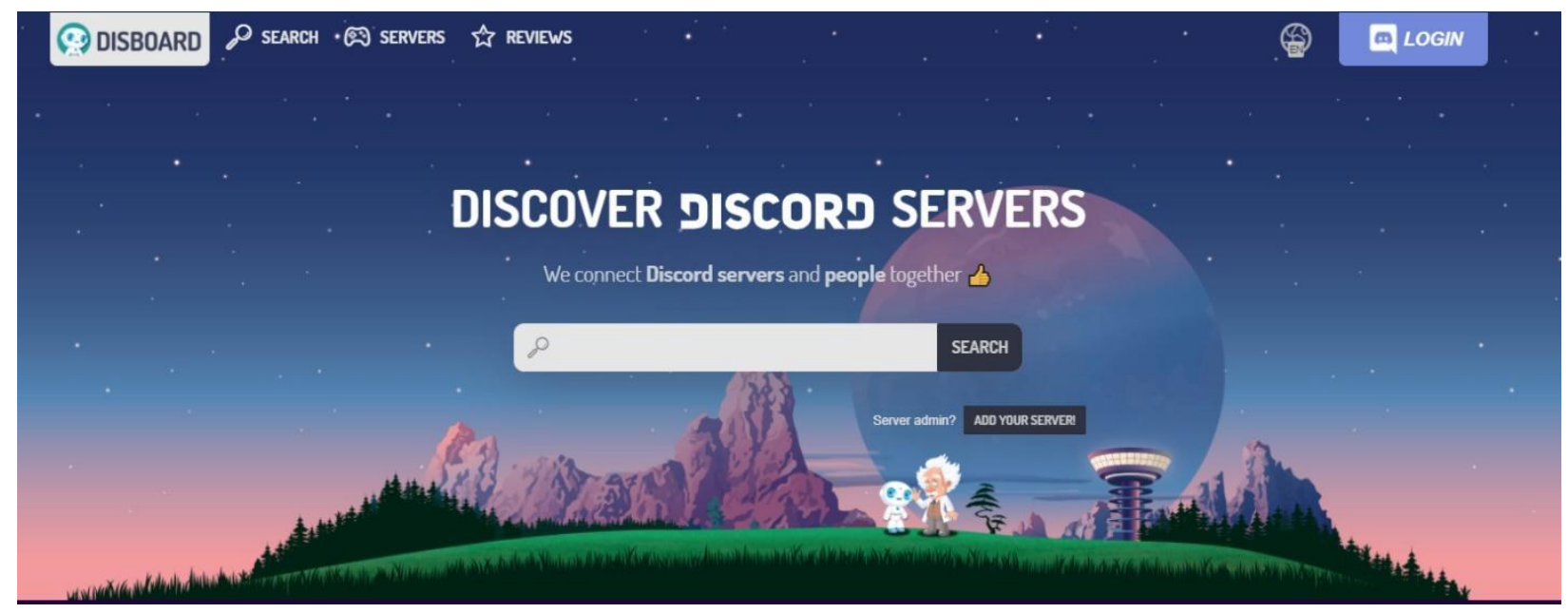

Figure 3. Disboard imitates the 'cuteness' of Discord.

Our findings indicate a disconnect between Discord's policies and the networked practices of hate groups. Instead of eliding the problematic structures that have emerged from Discord's user ecology, a meaningful intervention would involve 1) recognizing the role of third-party bots and bulletin sites in shaping Discord's culture 2) building and maintaining a comprehensive search feature that Discord can moderate and take responsibility for 3 ) acknowledging the proliferation of hateful communities and approaching bans from a networked perspective, ensuring that hate groups cannot easily reestablish themselves. Due to Discord's unique structure as isolated nodes of private communities, it may ultimately be successful promoting its curated, illusory userbase. However, without either acknowledging or distancing itself from its network of actual use, Discord will continue to host hate groups, and continue to undermine the inclusive future it actively markets.

\section{References}

Brock, A. (2018). Critical technocultural discourse analysis. New Media \& Society, 20(3), 1012-1030. https://doi.org/10.1177/1461444816677532

Brown, A. (2020, June 30). Discord Was Once The Alt-Right's Favorite Chat App. Now It's Gone Mainstream And Scored A New \$3.5 Billion Valuation. Forbes. https://www.forbes.com/sites/abrambrown/2020/06/30/discord-was-once-the-altrights-favorite-chat-app-now-its-gone-mainstream-and-scored-a-new-35-billionvaluation/ 
Brown Jr., J. J., \& Hennis, G. (2019). Hateware and the Outsourcing of Responsibility. In J. Reyman \& E. M. Sparby (Eds.), Digital Ethics: Rhetoric and Responsibility in Online Aggression. Routledge.

Burgess, J., \& Matamoros-Fernández, A. (2016). Mapping sociocultural controversies across digital media platforms: One week of \#gamergate on Twitter, YouTube, and Tumblr. Communication Research and Practice, 2(1), 79-96. https://doi.org/10.1080/22041451.2016.1155338

D'Ignazio, C., \& Klein, L. F. (2020). Data Feminism. MIT Press.

Discord Transparency Report: Jan-June 2020 / by Nelly / Discord Blog. (n.d.). Retrieved December 7, 2020, from https://blog.discord.com/discordtransparency-report-jan-june-2020-2ef4a3ee346d

DiscordFederation/DisboardScraper. (2020). [Python]. Federation of Discord Servers. https://github.com/DiscordFederation/DisboardScraper (Original work published 2019)

Gillespie, T. (2018). Custodians of the Internet: Platforms, Content Moderation, and the Hidden Decisions that Shape Social Media. Yale University Press.

Jiang, J. A., Kiene, C., Middler, S., Brubaker, J. R., \& Fiesler, C. (2019). Moderation Challenges in Voice-based Online Communities on Discord. Proceedings of the ACM on Human-Computer Interaction, 3(CSCW), 55:1-55:23. https://doi.org/10.1145/3359157

Massanari, A. (2017). \#Gamergate and The Fappening: How Reddit's algorithm, governance, and culture support toxic technocultures. New Media \& Society, 19(3), 329-346. https://doi.org/10.1177/1461444815608807

Noble, S. U. (2018). Algorithms of Oppression: How Search Engines Reinforce Racism. NYU Press.

Safety Principles and Policies. (n.d.). Discord. Retrieved April 8, 2021, from https://discord.com/safety 